\title{
Co-Creation Stage: A Web-Based Tool for Collaborative and Participatory Co-Located Art Performances
}

\author{
Héctor Rivas \\ hrivas@vicomtech.org \\ Vicomtech Foundation, Basque \\ Research and Technology Alliance \\ (BRTA) \\ San Sebastián, Spain \\ Iñigo Tamayo \\ itamayo@vicomtech.org \\ Vicomtech Foundation, Basque \\ Research and Technology Alliance \\ (BRTA) \\ San Sebastián, Spain \\ Alina Striner \\ alina.striner@cwi.nl \\ Centrum Wiskunde \& Informatica \\ Amsterdam, The Netherlands
}

\author{
Ana Domínguez \\ adominguez@vicomtech.org \\ Vicomtech Foundation, Basque \\ Research and Technology Alliance \\ (BRTA) \\ San Sebastián, Spain \\ Mikel Zorrilla \\ mzorrilla@vicomtech.org \\ Vicomtech Foundation, Basque \\ Research and Technology Alliance \\ (BRTA) \\ San Sebastián, Spain \\ Jie Li \\ jie.li@cwi.nl \\ Centrum Wiskunde \& Informatica \\ Amsterdam, The Netherlands
}

\author{
Stefano Masneri \\ smasneri@vicomtech.org \\ Vicomtech Foundation, Basque \\ Research and Technology Alliance \\ (BRTA) \\ San Sebastián, Spain \\ Pedro Almeida \\ almeida@ua.pt \\ University of Aveiro \\ Aveiro, Portugal
}

\begin{abstract}
In recent years, artists and communities have expressed the desire to work with tools that facilitate co-creation and allow distributed community performances. These performances can be spread over several physical stages, connecting them on real-time towards a single experience with the audience distributed along them. This enables a wider remote audience consuming the performance through their own devices, and even grants the participation of remote users in the show. In this paper we introduce the Co-creation Stage, a webbased tool that allows managing heterogeneous content sources, with a particular focus on live and on-demand media, across several distributed devices. The Co-creation Stage is part of the toolset developed in the Traction H2020 project which enables community performing art shows, where professional artists and nonprofessional participants perform together from different stages and locations. Here we present the design process, the architecture and the main functionalities of the tool as well as the results of the first user evaluation with Opera houses and artists.
\end{abstract}

\section{CCS CONCEPTS}

- Human-centered computing $\rightarrow$ Collaborative and social computing; Computer supported cooperative work; • Computer systems organization $\rightarrow$ Real-time systems.

Permission to make digital or hard copies of all or part of this work for personal or classroom use is granted without fee provided that copies are not made or distributed for profit or commercial advantage and that copies bear this notice and the full citation on the first page. Copyrights for components of this work owned by others than ACM must be honored. Abstracting with credit is permitted. To copy otherwise, or republish, to post on servers or to redistribute to lists, requires prior specific permission and/or a fee. Request permissions from permissions@acm.org.

IMX, 2021, NYC

(c) 2021 Association for Computing Machinery. ACM ISBN 978-1-4503-XXXX-X/18/06 ..\$15.00

https://doi.org/10.1145/1122445.1122456

\section{KEYWORDS}

Opera, WebRTC, Distributed performance

\section{ACM Reference Format:}

Héctor Rivas, Ana Domínguez, Stefano Masneri, Iñigo Tamayo, Mikel Zorrilla, Pedro Almeida, Alina Striner, Jie Li, and Pablo Cesar. 2021. Co-Creation Stage: A Web-Based Tool for Collaborative and Participatory Co-Located Art Performances. In IMX '21: ACM International Conference on Interactive Media Experiences, June 21-23, 2021, New York, NY. ACM, New York, NY, USA, 8 pages. https://doi.org/10.1145/1122445.1122456

\section{INTRODUCTION}

The co-creation and participatory art ideas are in the heart of fighting against social inequality, involving citizens in the creative process. The Traction project [9] promotes these collaborative and inclusive concepts for opera, a cornerstone of European cultural heritage. The project aims to empower people and communities in three trials (inner-city Barcelona, a youth prison in Leiria and diverse communities in Ireland) and to embrace new technologies to explore novel audio-visual formats.

The trial in Leiria, led by the artistic school SAMP, cooperates with a prison for young inmates and the aim is to lower the criminal recidivism rate by engaging the inmates in a collaborative and participatory opera process. For this trial, the opera will involve professional musicians and artists, inmates and their relatives, prison workers and ex-inmates all creating and acting together in a distributed performance.

In June 2021, a collaborative and participatory performances will be done, involving:

(1) A main stage in Lisbon, with professional artists and inmates from Leiria performing together, and physical audience in the theatre, 
(2) a stage in the prison in Leiria with professional artists and another group of inmates performing together, also with physical audience in the prison

(3) one or many homes, distributed across the world, with relatives of the inmates acting as remote viewers and participants of the performance.

In this article we present the Co-Creation Stage, a Web-based tool that enables distributed performances connecting different stages and people. We describe the user-centred design process followed to gather its requirements and provide the architectural design of the first release implementation.

Using the Co-creation Stage tool, the artists can create a template for their show, defining beforehand a number of scenes, the number of stages, the screens/displays/projectors/devices at each stage and audiovisual assets including live and pre-recorded content. During the show, thanks to a specific Web interface, an operator is able to manage the transition from one predefined scene to the next one, or modifying on-the-fly (if needed) all the settings according to the requirements of the performance. The operator, can also orchestrate the content to be shown on each output display, no matter if it is a screen on a stage or any display device used by a remote viewer or participant. Finally, the operator can also modify the layout template of each output display, providing the desired combination of assets on each screen. Fig. 1 provides a conceptual diagram of the capabilities of the tool.

\section{RELATED WORK}

According to [10], "orchestration" refers to the ability of a live video communication system to adapt in real-time to each context to enhance the quality of mediation and subsequently the quality of interaction between participants. In the case of Traction, applying it for community opera performances, this paradigm enables colocated performances with multiple voices on a single performance [6].

A tool like the Co-creation Stage should provide several key features:

- Orchestration: the ability of to automatically manage and distribute different contents across users, and support different type of devices and media contents;

- A Communication platform: to provide real-time notifications and synchronization between the end-users, enabling anyone to participate in the performance;

- Pre-production capabilities: it should offer basic pre-production functionality, such as the management of the overall timeline, spatial layout, and different types of sources and the effects;

- High quality, synchronized music transmission over the internet;

- Universality: to enable all participants (professional and non-professionals) participating through their own devices and/or professional material.

There are commercial tools on the market that fulfill one or more of these requirements, but none of the existing solutions is able to provide all the requested features. Jamulus ${ }^{1}$ and JamKazam

\footnotetext{
${ }^{1}$ https://jamulus.io/
}

2 provide the ability to play synchronized music across different remote locations; Parse ${ }^{3}$ and Firebase ${ }^{4}$ enable communication and synchronization functionalities through notification and WebRTC streams; Ableton Live ${ }^{5}$, OBS Studio ${ }^{6}$ and Max MSP $6{ }^{7}$ are production tools with a focus on music production; Carbyne ${ }^{8}$, BVMS ${ }^{9}$ (and again OBS Studio) provide orchestration functionalities. A tool that relies on OBS and WebRTC, OBS Ninja ${ }^{10}$ focuses on providing basic orchestration functionalities to web based conference calls. Fig. 2 compares the Co-creation stage with these tools in terms of the functionalities provided.

On the other hand, previous research projects have contributed to define and create innovative multi-screen services in the field of entertainment. For example, 2-IMMERSE [5] [11] is an opensource platform for object-based multi-screen entertainment where viewers can customise the content depending on their device, bandwidth and preferences. MediaScape [3] [13] aimed at providing interoperable technologies for the creation of HTML5-based media services that can be distributed across any type of connected devices. VConect project [12] explored distributed theatre performances connecting two stages from different locations. Online Orchestra [7] is a telematic performance project, aimed at enabling amateur musicians in remote locations to make music together over the Internet. [2] describes a pilot test on the sense of presence and quality of the interaction Networked Music Performance. [8] presents a platform for group communication that allows participants to create video communication session centred on a shared activity. Finally, [4] presents a script-based approach that provides multi-camera telepresence for distributed theatre.

All of these tools and projects provide very relevant features to enable distributed artistic performances, but none includes the whole range of features we are looking for. Accordingly, the Cocreation Stage has been created aiming at empowering professional artists and giving them the possibility to easily produce and run distributed events. This innovative tool, meets all the specified requirements in terms of production, orchestration and communication capabilities while guaranteeing high quality content and the universality that a Web-based solution provides.

\section{REQUIREMENTS GATHERING}

Before starting the definition of the design and architecture of the Co-Creation Stage, we conducted several focus group interviews with the artistic team of the TRACTION project, which includes two opera houses (Gran Teatre del Liceu in Barcelona and Irish National Opera in Dublin) and SAMP, an artistic school in Pousos (Portugal).

The focus group interviews were conducted as informal chats where the participants expressed their ideas about using technology. Then, the developers presented their vision about how to meet those ideas with a tool. Next, the discussion focused on the profiles who

\footnotetext{
${ }^{2}$ https://jamkazam.com/

${ }^{3}$ https://parseplatform.org/

${ }^{4}$ https://firebase.google.com/

${ }^{5}$ https://www.ableton.com/en/live/

${ }^{6}$ https://obsproject.com/

${ }^{7}$ https://cycling74.com/

${ }^{8}$ https://carbyne911.com/

${ }^{9}$ https://www.boschsecurity.com/us/en/solutions/management-software/bvms/

${ }^{10}$ https://github.com/steveseguin/obsninja
} 


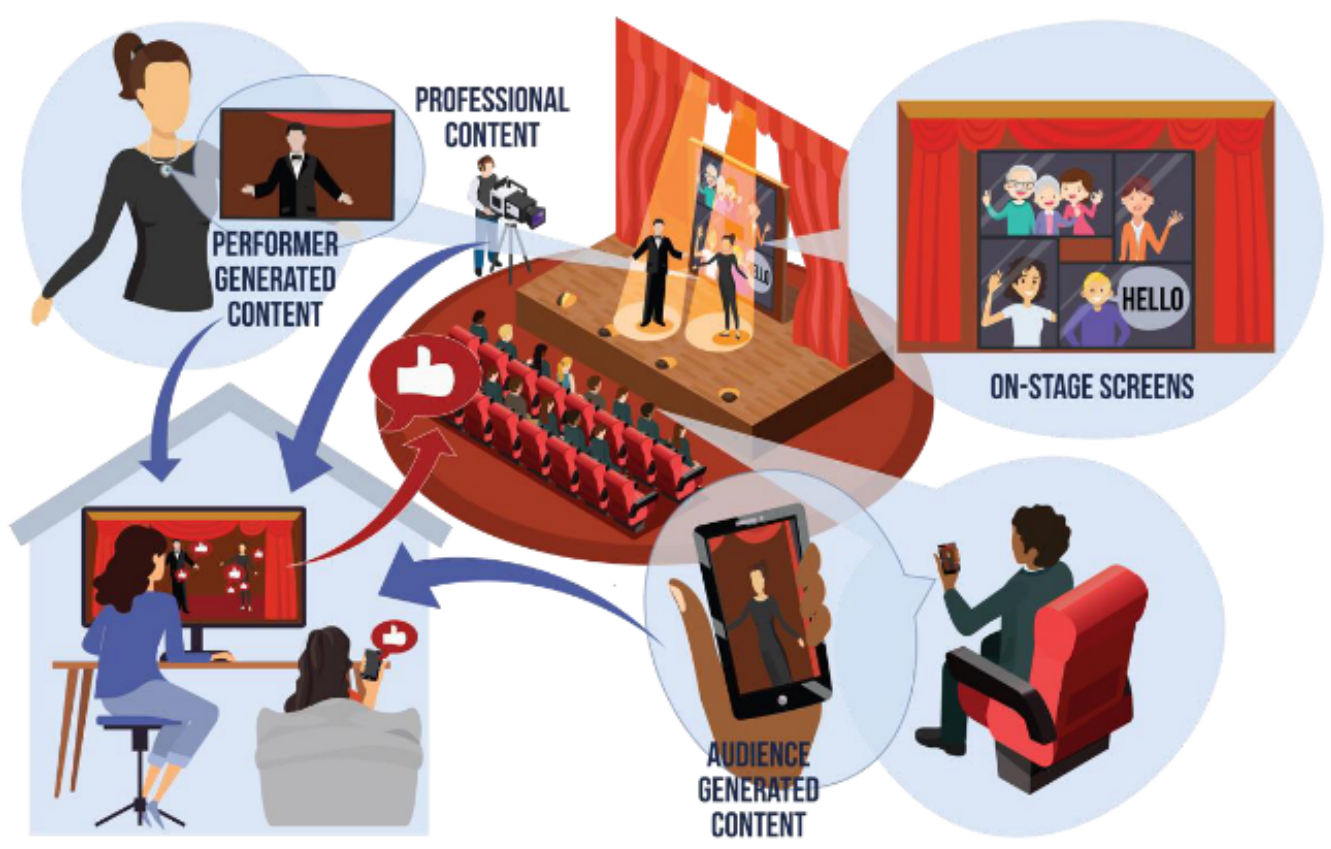

Figure 1: The Co-creation Stage is a tool that enables distributed performances.

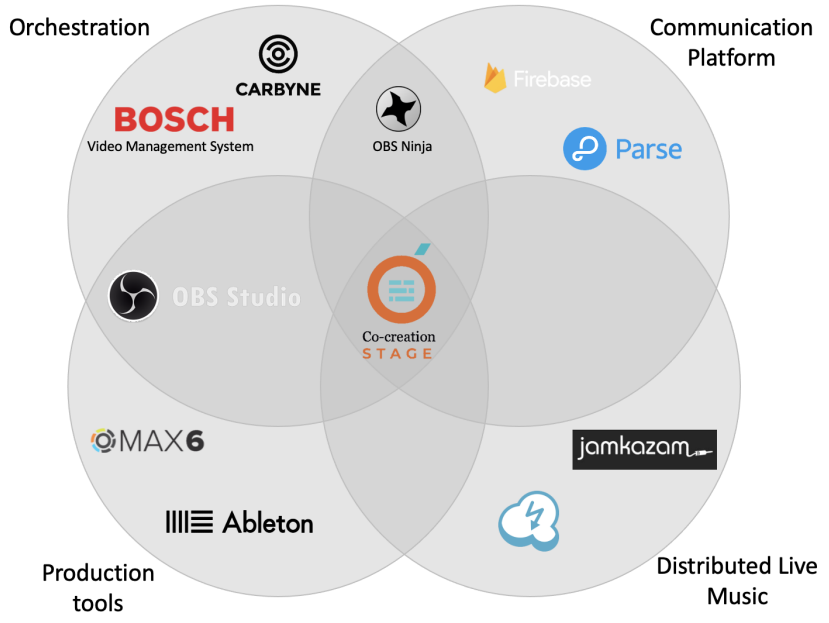

Figure 2: Comparison of the Co-creation Stage with existing tools

would be using the Co-Creation Stage and how the tool could help the artistic process in the participatory performance phase.

The artistic team defined a very concrete set of requirements and described how, over 2021 and 2022, they would define several pilot performances to get familiar with the Co-Creation Stage interface and have different stakeholders use it. The tool would also be used in rehearsals to enable the participation of remote users, for example, that were not able to meet in person due to Covid-19 or to restrictions in the prison. The artistic team mentioned several times that the tool should be flexible, allowing the director to manage unscripted content and being able to deal with issues that may occur during a live performance. Another request was about the interface of the Co-Creation Stage: it could be complex for the creation of an opera template, but it should be very intuitive when used during the live representation. Moreover, it should promote universality, allowing the use of professional equipment (cameras, microphones, audio cards, projectors, etc.), but also the inclusion of non-professional participants with their own devices (such as the mother of an inmate with a mobile phone).

The focus groups were analysed using thematic analysis [1]: the audio of the interviews was transcribed and coded by two researchers, which reached an agreement for the high level themes emerging from the data. Table 1 summarises the requirement types collected during the Focus Group and the thematic analysis.

\section{ARCHITECTURE DESIGN AND IMPLEMENTATION}

This section describes the architecture of the Co-Creation Stage as well as a detailed description of each component and their interfaces.

\subsection{Architecture of the Co-creation Stage}

The Co-Creation Stage is based on a client-server architecture on top of Web technologies and uses a platform that allows the orchestration, communication and distribution of content between different devices and multi-device interaction. Fig. 3 shows the architecture of the Co-Creation Stage. 
Table 1: Co-Creation Stage requirement types

\begin{tabular}{ll}
\hline Code Type & Requirement \\
\hline Communication & Flexibility; helpful in co-creation settings; allowing bi-directional communication between remote \\
& users and people in the prison. \\
Support for multiple users; easy to use for non-technical people, availability of keyboard short- \\
cuts and video tutorials and promoting universal access for professional and non-professional \\
equipment. \\
Support for pre-recorded content; focus on high-quality audio; support uploading and displaying \\
of image slideshows, videos, audio tracks.
\end{tabular}

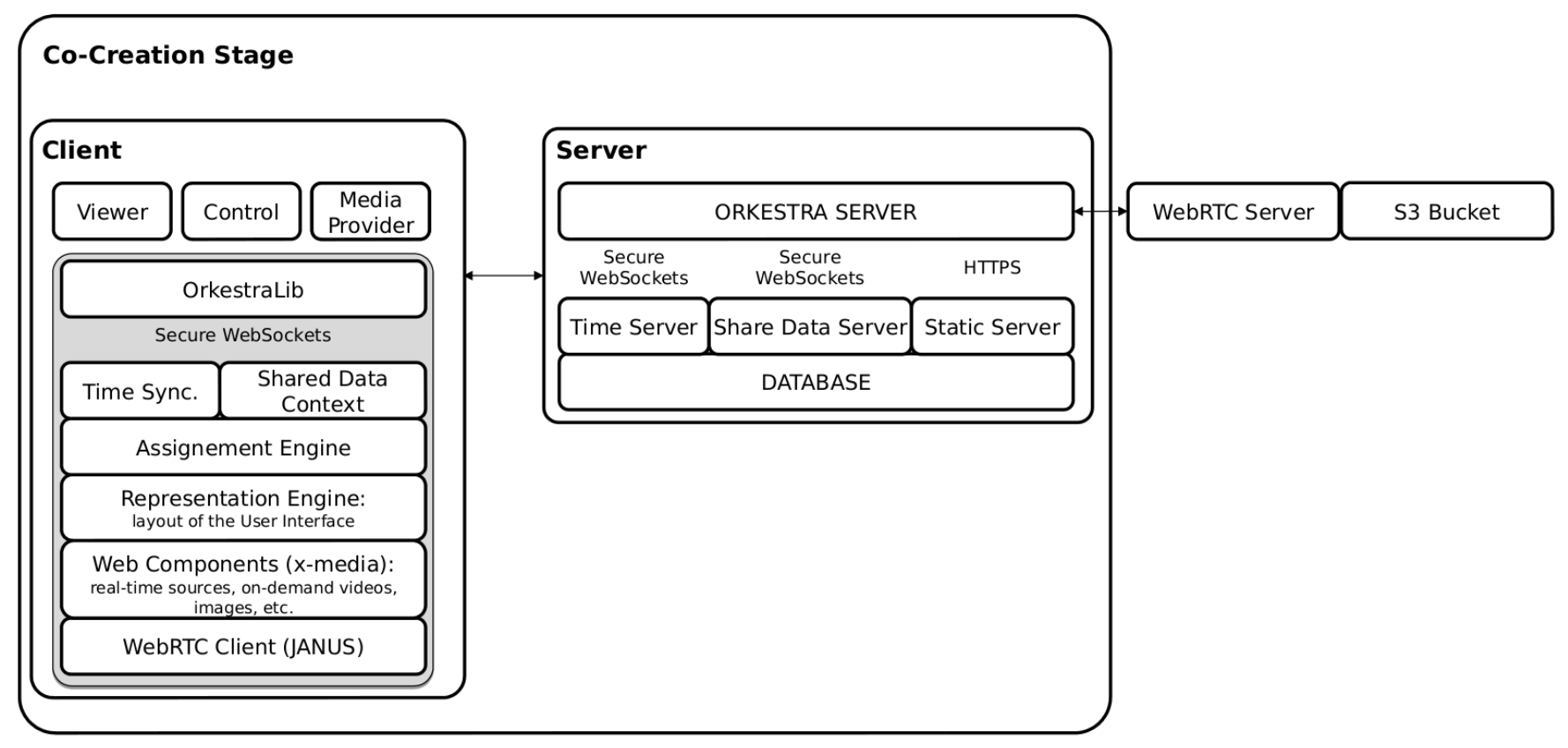

Figure 3: Co-Creation Stage architecture

The Co-creation Stage is therefore made of two components: the client side composed of three applications (Control, Viewer and Media Provider) and a server-side implementation. The latter is composed by several modules:

- A Rest API for the manipulation of data persistence;

- A Websocket Server, for sharing the context of the application in real-time;

- A Timing Server for sharing a timer which is synchronized to millisecond accuracy;

- A WebRTC gateway that allows publishing and consuming real-time media flows.

The client side contains most of the application logic, distributed in its three applications:

- The Control application provides the interface for the operator allowing to create the event, to manage the sources (cameras and videos) that it will contain, as well as the devices that will show the content. It is also able to customise the layout template that each device will show and act on them in real-time

- The Viewer is a simpler application for visualising the content on each display according to the orchestration decided by the operator.

- The Media Provider offers the functionalities required to integrate the live audio and/or video streams.

The Control application is based on a user interface that allows the operator to manage the rooms (stages, locations, etc.) and their devices (displays or projectors on a stage, a mobile device, etc.), the role of each device (viewer and/or media providers), and the multimedia assets. The operator can create a timeline structure that defines which components are associated to which sources and how they will be represented in each device on each location. This information is shared in real time with each connected device through a Shared Context. Furthermore, there is a timeline timestamp to synchronise all the content which is propagated using Timing Server. 
The Viewer is build on top of media components. According to the orchestration defined by the Control, a viewer visualises $N$ media components, creating a specific layout assigning size and location to each of the components. Each media component is able to render real-time streams through WebRTC, on-demand videos (such as mp4 files, HLS or MPEG-DASH), images, audios, etc.

\subsection{User Interfaces of the Co-creation Stage}

As explained in the previous subsection, the Co-creation Stage is composed by three applications and interfaces at the client side: the ones for the Control, the Viewer and the Media Provider.

The Control application allows the artists to see all the generated shows (Fig. 4A); to create a template for their show and define a number of scenes in a timeline (Fig. 4B); to specify the number of locations that will be used as well as devices for each location and their role (viewer and/or media provider) (Fig. 4C); and to load pre-recorded media assets (Fig. 4D).

During the show (Fig. 5 Theatre, represents the main stage with a musician and two projectors), an operator is able to fully control the Co-creation Stage and modify on-the-fly all the settings through a specific interface (Fig. 6). This interface is divided in five main parts. In the Media section, all the available on-demand assets are shown as a preview. There is also a Cameras section, where all the defined live cameras preview coming from the Media Providers are shown. The Preview section allows the operator to see what is being displayed at each display in each room. At the Components section, the operator can assign a specific component to a row (an on-demand video or a real-time stream). Finally, in the Timeline, where each column represents a scene, the operator selects the components to be shown (the selected green boxes where the audio can be activated or not) and a User Interface layout is selected for each scene.

In Fig. 5, Remote participant, an artist can remotely participate in the show, using a tablet as a media provider and a viewer, following the show in the main stage, and being part of the show across one of the projectors in the theatre. In Fig. 5, Remote viewer, a remote viewer can follow the whole show from a tablet. The operator orchestrates all the experience, deciding what to show for the remote viewers, the remote participants and the displays on the stages, moving between scenes and deciding the layout of each output display.

Remote users will follow the show through the Viewer application. When the application is opened in a web browser, an interaction is needed to be able to manage the media of the browser. Once the user presses the start button (Fig. 7A), the content is shown in full screen according to the operator decisions (Fig. 7B shows a layout with four on-demand media assets).

Figure 8 shows the interface for the media provider, where the users just need to authorise the use of the camera and the microphone from the Web browser and select the specific device (camera and microphone) they intend to use. From that moment on, the camera is enabled and ready for the operator.

\section{USER TESTS AND EVALUATION}

The first release of the Co-Creation Stage was tested in the Leiria (Portugal) young correctional facility, where SAMP is leading the co-creation of novel community opera capsules.

The evaluation was conducted by performing 2 user test sessions complemented by a discussion with 4 SAMP members, and the interviews were conducted in Portuguese. Even though a larger number of end-users where scheduled, we finally managed to test the system with two of them due to Covid-19 restrictions.

For the user tests, we devised a session composed of 8 tasks, each one requiring about five minutes to complete. After each task, the user had to answer a Single Ease Question (SEQ) to rank, in a range from 1 to 7 , the difficulty of the task (1 representing "very difficult" and 7 "very easy"). After the completion of the 8 tasks the user had to fill a System Usability Scale (SUS) questionnaire and answer a set of open questions about the experience of using the Co-Creation stage to complete the tasks.

For the tests in the prison, Internet connectivity was provided by a $4 \mathrm{G}$ router. The materials used to run the tests were the following:

- One PC with an Nvidia graphic card and a Blackmagic sound card;

- One Canon camera;

- Two projectors, each one connected to an Intel NUC;

- Two mobile devices.

The tests were run in two separate rooms inside the prison, one acting as the main stage (where the main PC and projectors were installed) and another room to simulate the connection of a remote stage. The devices were not in the same LAN/WLAN, they were connected to the Internet to access the server-side elements, deployed over an AWS instance. The testers were asked to complete the following tasks:

(1) Check live cameras in the stage and start the displays acting as viewers;

(2) Check connection from remote user acting as media providers;

(3) Start the performance;

(4) Navigate from one scene to the next;

(5) Browse until the end of the performance;

(6) Change the layout of a display;

(7) Display a different component on a specific scene for a particular display on the main stage;

(8) Load an existing pre-defined template with another schedule for the show.

The types of findings we expected to get from the user tests were about usability issues, additional requirements or about the value of the Co-creation Stage as a tool to enable collaborative and participatory co-located art performances. All the users agreed on the fact that the system was user friendly and in general easy to use. Task 7 was the one considered the most difficult one by the users, but the average rating for the SEQs was 6.4, very close to the maximum of 7 . The users agreed on the usefulness of the Co-creation Stage as a tool for enabling distributed performances, but they also identified some usability issues (summarised in Table 2) that were used to define, together with the feedback from the artistic team, the requirements for the next iteration of the tool. 


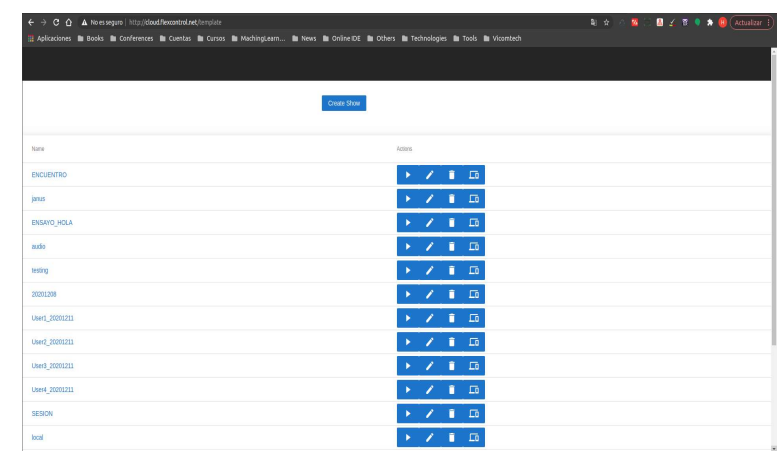

(A)

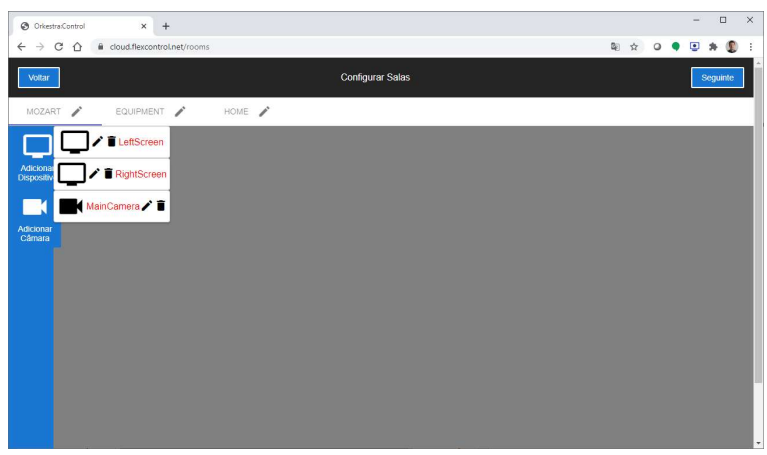

(C)

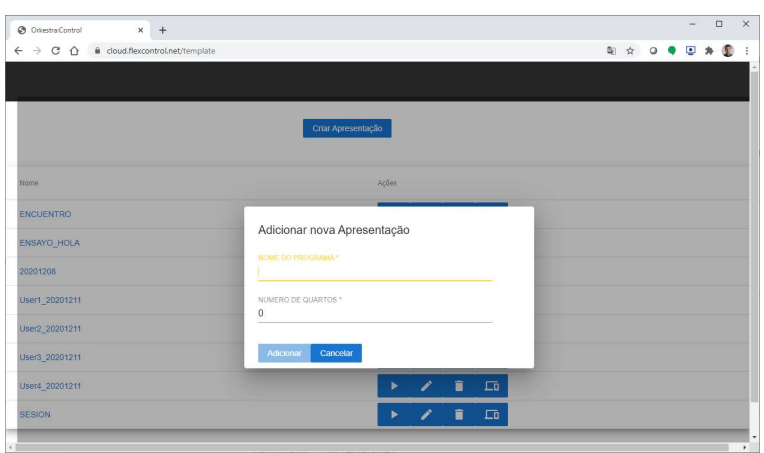

(B)

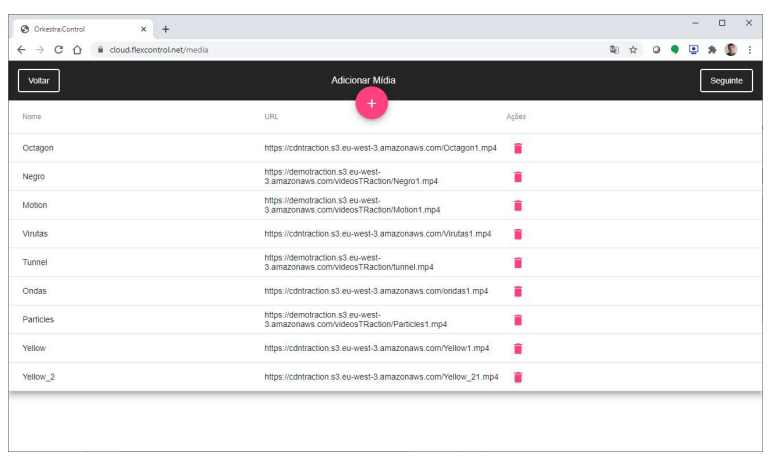

(D)

Figure 4: Template generation interfaces for the configuration of an event
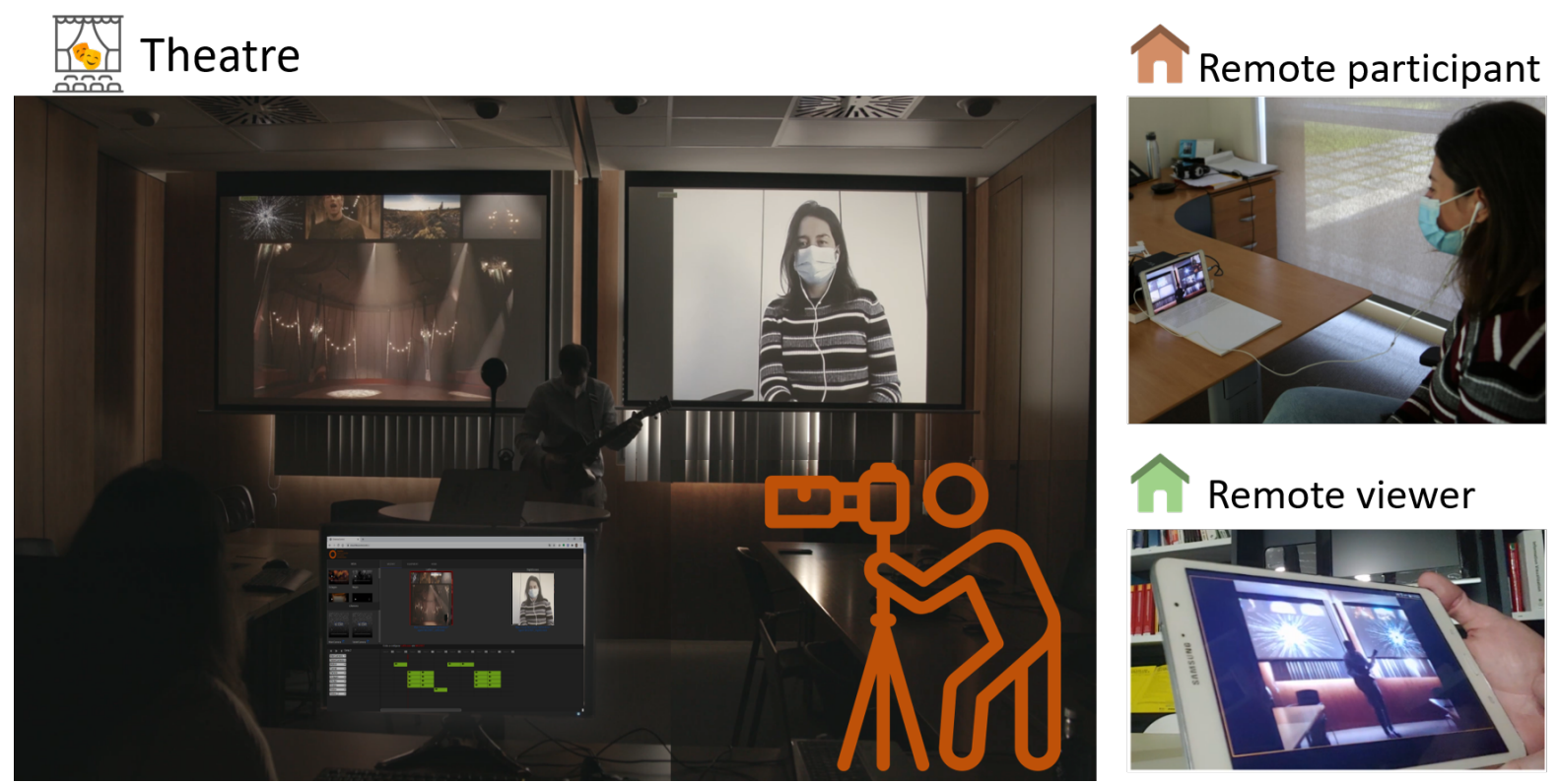

Figure 5: Overview of the Co-creation Stage in a lab environment. 


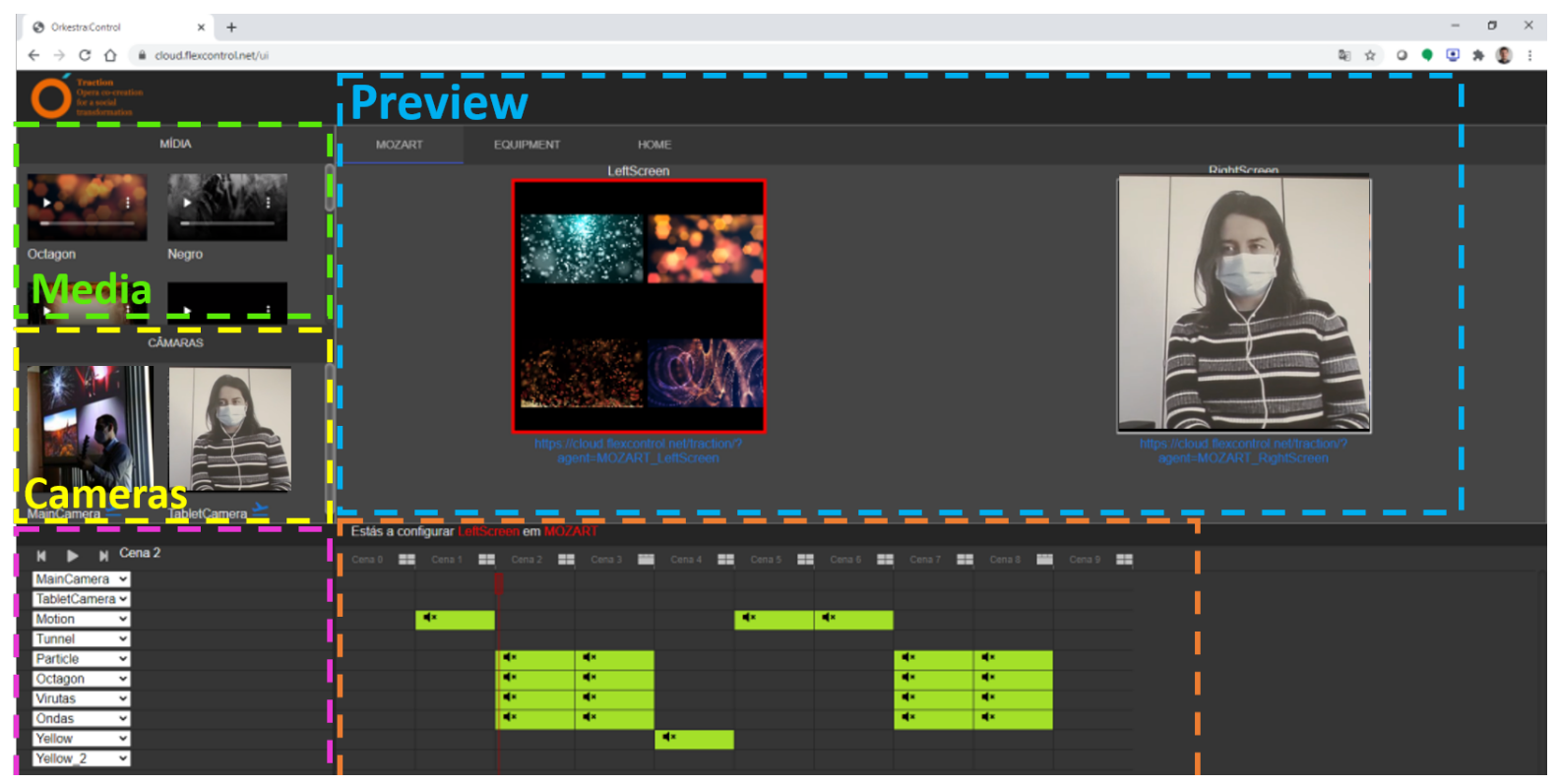

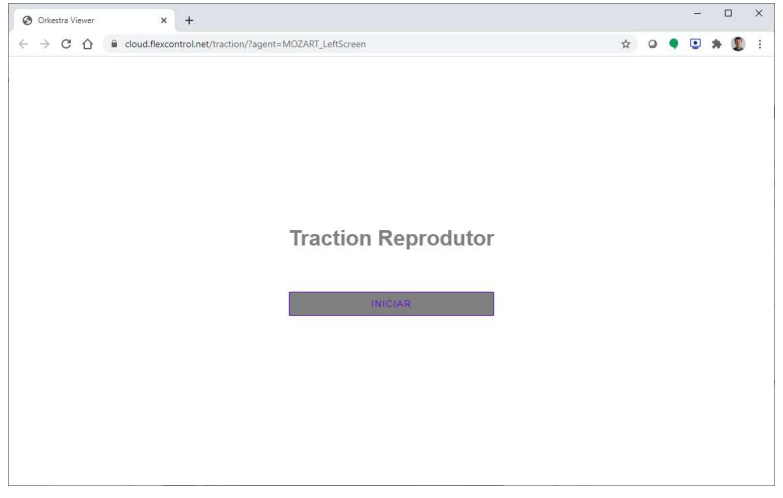

(A)

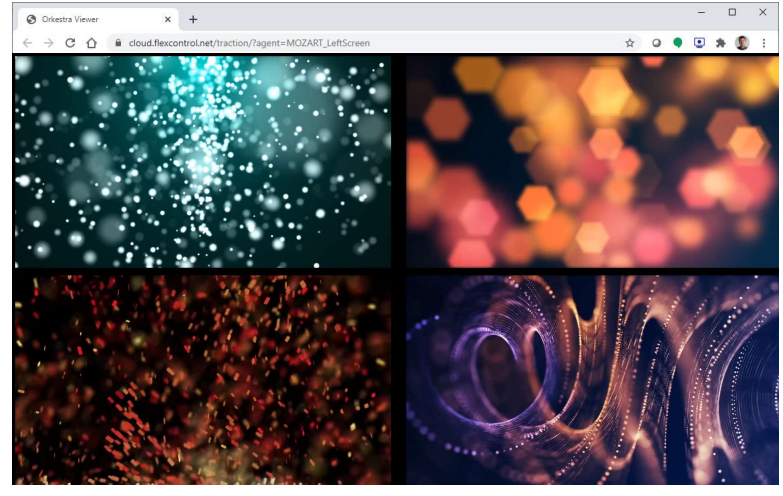

(B)

Figure 7: Interface of the Viewer application. The users only has to press the start button (A) to join the session and see the content according to the operator decisions (B).

\section{CONCLUSIONS AND FUTURE WORK}

This article presents the Co-creation Stage, a tool enabling participatory distributed art performances. This paper describes the User-Centred Design process followed with the artistic teams participating in the Traction H2020 project to gather the requirements and propose the design of the tool. A first release of the tool has been implemented and user tests have been done to evaluate the UI/UX and gather insights from the stakeholders. The preliminary results provide positive indications towards the use of such a tool in collaborative and participatory co-located art performances.

The next steps are to incrementally improve the Co-creation Stage interface and functionalities based on the feedback gathered during the user tests and new focus groups under the second iteration of the User-Centred Design process. Moreover, the Co-creation Stage tool will be used in several performances, such the aforementioned co-located performance in Portugal in June 2021 and more 
Table 2: Usability issues identified through user tests

\begin{tabular}{ll}
\hline Type & Issue \\
\hline UI & The process for changing scene is not intuitive. \\
UI & It's difficult to replace a component with a media that is not already in the timeline. \\
UX & There are no options such as pausing videos or controlling volume. \\
UX & Some of the icons do not represent the button behaviour. \\
UX & Replacing one component in the timeline replaces it in every scene instead that only in the current scene. \\
UX & Sound quality needs improvement, since the media providers and WebRTC are oriented for videocalls and not \\
& for music, applying noise suppression, echo cancellation and other filters. \\
\hline
\end{tabular}

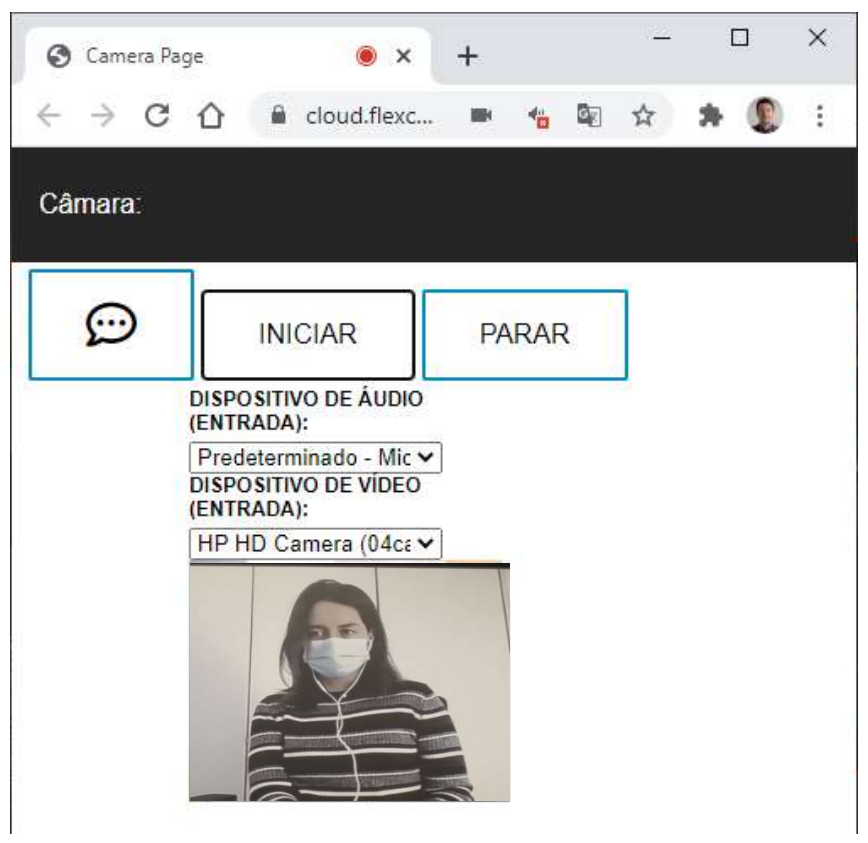

Figure 8: Interface of the Media provider application

participatory performances both in Portugal and Barcelona during 2022.

\section{ACKNOWLEDGMENTS}

The research in Vicomtech and CWI has been supported by European Union's Horizon 2020 research and innovation programme under grant agreement No 870610, project Traction (Opera cocreation for a social transformation). The authors would like to thank Loinaz Merino for acting as the remote participant in the lab environment deployment (Fig. 5, 6, 8).

\section{REFERENCES}

[1] Virginia Braun and Victoria Clarke. 2012. Thematic analysis. American Psychological Association, Washington D.C., USA, 57-71.

[2] Stefano Delle Monache, Michele Buccoli, Luca Comanducci, Augusto Sarti, Giovanni Cospito, Enrico Pietrocola, and Filippo Berbenni. 2018. Time is not on my side: network latency, presence and performance in remote music interaction. In Proceedings of the XXII CIM Colloquium on Music Informatics-Machine Sounds, Sound Machines. DADI - Dip. Arti e Design Industriale. Università IUAV di Venezia, Udine, Italia, 152-158.
[3] Ana Dominguez, Mikel Agirre, J Flörez, Alberto Lafuente, Iñigo Tamayo, and Mikel Zorrilla. 2017. Deployment of a hybrid broadcast-Internet multi-device service for a live TV programme. IEEE Transactions on Broadcasting 64, 1 (2017), 153-163.

[4] Rene Kaiser, Marian F. Ursu, Manolis Falelakis, and Andras Horti. 2015. Enabling Distributed Theatre Performances through Multi-Camera Telepresence: Capturing System Behaviour in a Script-Based Approach. In Proceedings of the 3rd International Workshop on Immersive Media Experiences (Brisbane, Australia) (ImmersiveME '15). Association for Computing Machinery, New York, NY, USA, 21-26. https://doi.org/10.1145/2814347.2814351

[5] Ian Kegel, James Walker, Mark Lomas, Jack Jansen, and John Wyver. 2017. 2IMMERSE: A Platform for Orchestrated Multi-Screen Entertainment. In Adjunct Publication of the 2017 ACM International Conference on Interactive Experiences for TV and Online Video (Hilversum, The Netherlands) (TVX '17 Adjunct). Association for Computing Machinery, New York, NY, USA, 71-72. https://doi.org/10.1145/ 3084289.3089909

[6] François Matarasso. 2019. A restless art. How participation won, and why it matters. Digital edition. London: Calouste Gulbenkian Foundation. Viitattu 15 (2019), 2020.

[7] Michael Rofe, Samuel Murray, and Will Parker. 2017. Online Orchestra: Connecting remote communities through music. Journal of Music, Technology \& Education 10, 2-3 (2017), 147-165.

[8] Tim Stevens, Ian Kegel, Doug Williams, Pedro Torres, Pablo Cesar, Phil Stenton, Rene Kaiser, Marian Ursu, Manolis Falelakis, and Nikolaus Färber. 2012. Video communication for networked communities: Challenges and opportunities. In 2012 16th International Conference on Intelligence in Next Generation Networks. IEEE, IEEE, Berlin, Germany, 148-155. https://doi.org/10.1109/ICIN.2012.6376018

[9] Traction project members. 2021. TRACTION. https://www.traction-project.eu. [Online; accessed 18-March-2021].

[10] Marian F. Ursu, Manolis Falelakis, Martin Groen, Rene Kaiser, and Michael Frantzis. 2015. Experimental Enquiry into Automatically Orchestrated Live Video Communication in Social Settings. In Proceedings of the ACM International Conference on Interactive Experiences for TV and Online Video (Brussels, Belgium) (TVX '15). Association for Computing Machinery, New York, NY, USA, 63-72. https://doi.org/10.1145/2745197.2745211

[11] James Walker, Doug L Williams, Ian C Kegel, Andy P Gower, Jack Jansen, Mark Lomas, and Stefan Fjellsten. 2019. 2-IMMERSE: A Platform for Production, Delivery, and Orchestration of Distributed Media Applications. SMPTE Motion Imaging fournal 128, 7 (2019), 45-51.

[12] Douglas L. Williams, Ian C. Kegel, Marian Ursu, Pablo Cesar, Jack Jansen, Erik Geelhoed, Andras Horti, Michael Frantzis, and Bill Scott. 2015. A Distributed Theatre Experiment with Shakespeare. In Proceedings of the 23rd ACM International Conference on Multimedia (Brisbane, Australia) (MM '15). Association for Computing Machinery, New York, NY, USA, 281-290. https://doi.org/10.1145/ 2733373.2806272

[13] Mikel Zorrilla, Njål Borch, François Daoust, Alexander Erk, Julián Flórez, and Alberto Lafuente. 2015. A web-based distributed architecture for multi-device adaptation in media applications. Personal and Ubiquitous Computing 19, 5 (2015), $803-820$. 